\title{
‘Masked' communication
}

\section{Jane Merivale}

Senior Dento-legal Advisor for BDA Indemnity

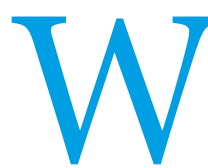

hilst masks can provide a barrier to droplets and the transmission of an airborne disease like COVID-19, the continuous use of masks poses both practical and ethical challenges for the dental team that are being reflected in an increasing number of complaints being received around the country. Some patients are complaining that their dentist was uncaring and indifferent to their pain. Others are saying that they have not understood the treatment options described by the clinician or the likely outcome.

Much has been written on how masks and full PPE can impact the clinician but, so far, less is known about the impact on patients attending for treatment.

Clearly, masks make communication more difficult and create a psychological barrier to the development of a robust and professional therapeutic relationship that is crucial for the development of trust and mutual understanding. Dentistry requires a degree of collaboration and understanding between the patient and dentist if the process of decisionmaking is to be effectively shared. Any barrier to communication in the dental setting will diminish the level of rapport and increase the potential for patient dissatisfaction.

\section{Masked for the whole appointment}

At the time of writing, the standard

Operating Procedure (SOP) requires the clinical team to wear masks not only for the operative procedure but for the whole of the interaction with the patient. To the patient, one clinician soon becomes indistinguishable from another.

Masks intended to protect a patient's safety, may also seem threatening and hence create additional anxiety and distress in a patient already worried about dental treatment. Mask wearing of course is familiar, however where previously we might have pulled down our mask, turned to face our patient to make eye contact and smile reassuringly, this is currently not possible. We need to enhance other means of communication to compensate for the loss of visible facial expression.

\section{What is missing?}

Facial expressions signal our thoughts and emotions and most of us become fairly adept at reading the faces of others to understand what they are thinking and feeling. We have evolved 42 muscles of facial expression for this purpose!

Being able to observe the mouth can impact on the patient's perception of a dentist's emotional intelligence, and their likeability, which directly affects patient satisfaction, regardless of the actual treatment outcome.

\section{Develop these skills to compensate} for any reduction in effective communication when wearing a mask

$\rightarrow$ The environment - Minimise the noise and distractions in the surgery; patients need to understand what is being said and if not hearing fully, especially in the absence of lip-reading cues, they will 'make a guess' at what's been said, particularly patients with a cognitive or hearing impediment

$\rightarrow$ Make eye contact - This conveys 'I see you' activating empathy and connection. Too much and the patient feels uncomfortable, but enough strengthens the greeting and promotes trust

$\rightarrow$ Introductions are key - Wear a name badge so everyone knows 'who's who' and their job title

$\rightarrow$ Explain why you are wearing a mask This can enhance trust in the dental setting signalling adherence to cross infection control measures given dentistry is carried out at such close quarters

$\rightarrow$ Listen well - Let patients tell their story, uninterrupted

$\rightarrow$ Give reassurance That the patient is safe and acknowledge the extra difficulties imposed by wearing a mask

$\rightarrow$ Check your tone of voice - The tone conveys over $38 \%$ of the non-verbal emotional content of what we say. The pace, rhythm and pitch of spoken language is called prosody. Prosody infuses a layer of emotion that goes above and beyond the singular meaning of each word and we are all highly sensitive to variations in tone of voice. In a famous study by Nalini Ambady, ${ }^{1}$ audiotapes of surgeons talking with patients were filtered so only the volume, pace and rhythm of their communications were audible. When the tapes were played to a group of volunteers, listeners could determine the surgeons who had a history of complaints and claims

$\rightarrow$ Name your emotion - If the PPE makes it difficult to express it: for example, 'You make me smile' or 'I empathise with you'

$\rightarrow$ Convey openness, warmth and respect with body language - Sit down with patients, turn towards them, and sit at eye level whilst maintaining social distance.

$\rightarrow$ Encourage questions to gauge understanding - In the face of any lack of comprehension that is critical to obtaining valid consent. Information gathered by the dentist may otherwise be incomplete leading to clinical and consent inaccuracies.

$\rightarrow$ Use gestures - Thumbs up or down to clarify what has or hasn't been understood

$\rightarrow$ Give more supplementary written information than usual

$\rightarrow$ Safeguard confidentiality - It can be difficult to speak louder for clarity. You may have to move somewhere more confidential if the situation demands it

$\rightarrow$ Use technology - Creatively to supplement information given - some dentists are experimenting with live transcript applications compatible with mobile phone technology as a means of communication solving, so the patient can listen again when they've left the surgery.

\section{Conclusion}

Brush up your communication skills. There has never been a time when enhanced communication efforts and the conveyance of empathy has been more important. The use of a mask creates the need to really work on communication skills to create trust and enhance patient care.

\section{Reference}

1. Ambady N, Laplante D, Nguyen, T et al. Surgeons' tone of voice: a clue to malpractice history. Surgery 2002; 132: 5-9.

https://doi.org/10.1038/s41404-021-0765-9 